Archives de sciences sociales des religions

111 | juillet-septembre 2000

Varia

\title{
Des formes ordinaires de la vie religieuse
}

Entre anthropologie et ethnographie

\section{Albert Piette}

\section{CpenEdition}

\section{Journals}

Édition électronique

URL : http://journals.openedition.org/assr/20230

DOI : $10.4000 /$ assr.20230

ISSN : $1777-5825$

Éditeur

Éditions de l'EHESS

\section{Édition imprimée}

Date de publication : 1 septembre 2000

Pagination : 125-133

ISBN : 2-222-96695-7

ISSN : 0335-5985

\section{Référence électronique}

Albert Piette, "Des formes ordinaires de la vie religieuse », Archives de sciences sociales des religions [En ligne], 111 | juillet-septembre 2000, mis en ligne le 19 août 2009, consulté le 03 mai 2019. URL: http://journals.openedition.org/assr/20230 ; DOI : 10.4000/assr.20230

Ce document a été généré automatiquement le 3 mai 2019.

(c) Archives de sciences sociales des religions 


\section{Des formes ordinaires de la vie religieuse}

Entre anthropologie et ethnographie

\section{Albert Piette}

1 Cet article ${ }^{1}$ a pour point de départ les difficultés et les résistances de la sociologie des religions à regarder le fait religieux tel qu'il se fabrique, et se produit au jour le jour dans la concrétude des diverses situations. Elle est plus tentée par le compte rendu des effets collectifs des interactions locales, que par la description des situations circonstancielles d'où ils surgissent. Pourquoi éliminer les circonstances, les hésitations des hommes, les faits ordinaires alors que ce sont eux qui font et construisent les pratiques dont les effets servent de support aux grandes interprétations ou théories macrosociologiques? Si ce diagnostic ne vaut pas seulement pour la sociologie du religieux, il nous semble que celleci cumule les difficultés générales de la sociologie à pratiquer une observation rapprochée de la vie sociale et celles spécifiques au fait religieux lui-même. C'est bien une ethnographie des formes ordinaires de la vie religieuse qui est en jeu.

\section{Le statut de l'observation en sciences sociales du religieux}

2 Un argument confortant la pratique de l'observation en marge de la sociologie du fait religieux réside dans les grandes inspirations théoriques de celle-ci : Durkheim, Weber ou encore Marx et Bourdieu, dont le goût pour les détails du quotidien est loin d'être évident (Piette, 1996a, ch. 1).

3 Ainsi Durkheim, qui associe le fait social à « une sorte de consistance " des manières d'agir ou de penser, constitutive d'«un produit nouveau» et isolé «des événements particuliers qui les reflètent ", ne donne aucune crédibilité à la méthode d'observation. Le face à face avec le réel que celle-ci suppose ne peut que faire voir cette consistance à l'état impur, c'est-à-dire imprégnée des particularités des individus qui la constituent. Il importe donc de « dégager le fait social de tout alliage pour l'observer à l'état de pureté » 
(Durkheim, 1993). D'où bien sûr la valorisation méthodologique des «variations concomitantes » et les recherches quantitatives et statistiques de moyennes. Exception : les «moments magiques» que constituent les manifestations rituelles comme moments maximisant la conscience collective et l'intériorisation des normes sociales. Dans les descriptions parfois absurdes qu'en donne Durkheim, toute infiltration individuelle et particulière y serait éliminée ; la réception y serait pure. L'individu y est noyé dans une homogénéité socioculturelle et un absorbement émotionnel impossibles.

Le renversement radical qu'oppose Weber à cette perspective ne modifie pas le statut de l'observation. Ce qui intéresse le sociologue allemand, c'est bien le sens visé par l'agent, tel qu'il oriente son comportement par rapport à autrui. L'activité de l'individu influencée et noyée dans une norme n'est plus considérée comme sociale, alors qu'elle était chez Durkheim le prototype même de l'action sociale. Weber analyse l'activité sociale en tant qu'elle renvoie aux catégories de «fin» et de «moyens» : se donner un but, établir des moyens propres, déterminer les conséquences, «comme si l'activité se déroulait effectivement avec la conscience de son orientation significative » (Weber, 1971 ). Ainsi Weber utilise la méthode idéaltypique pour cerner les «bonnes raisons » que les hommes ont d'accomplir telle ou telle action. Pour Weber, l'idéaltype qui est une « utopie » et non un " exposé du réel » est construit par l'accentuation de certains traits de la réalité (Weber, 1965 ; Piette, 1996a). Plus que l'observation, c'est donc le rapport rétrospectif (sous forme d'entretiens) qu'en font les individus qui intéresse Weber en rupture avec la réalité concrète des situations. L'homme moyen y est remplacé par l'homme typique, l'absorbement émotionnel par l'absorbement rationnel.

Que le chercheur ait envie de sortir du "Durkheim-Weber" pour préférer Marx ou Bourdieu, l'intérêt de l'observation sera tout aussi réduit puisqu'il s'agit là d'une volonté explicite de mettre entre parenthèses les aspects visibles de la réalité pour associer l'objet sociologique à une structure objective cachée, de telle sorte que l'action des hommes y est décrite comme une ombre par rapport à la présence agissante de forces et de déterminations sociales diverses.

Et l'ethnologie? pourrait objecter le lecteur. Il faut reconnaître que le religieux a subi, sauf rares exceptions, la dure loi du «Grand Partage » réservant le statut d'objet ethnologique aux formes "excentrées" du religieux (de la mythologie dogon à la religiosité populaire...) plutôt qu'aux formes «ordinaires" ou "courantes» du christianisme occidental, comme si celles-ci étaient privées des bizarreries exotiques chères aux ethnologues. Bref, l'ethnologie a désormais envahi les centrales nucléaires, les laboratoires scientifiques ou le Parlement européen mais pas encore vraiment l'administration diocésaine, la vie du prêtre ou du pasteur, les sessions synodales... Par ailleurs, si nous plaidons fortement pour que l'ethnologie «fourre son nez partout » et "se joigne à ce qui se passe" dans ces différentes situations (selon les mots de Malinowski), il faut d'emblée percevoir les limites de l'observation ethnologique dans sa version classique exercée en terrains " exotiques ». Comme nous l'avons montré ailleurs (Piette, 1996a), l'activité même du regard n'y est pas valorisée ni systématique. L'ethnologue a choisi une large unité d'observation : la culture qu'il ne peut cerner qu'à travers un regard distant par rapport à l'action en train de se faire, et mobile, toujours en déplacement dans l'unité territoriale choisie. Malinowski est clair à ce sujet: «il faut poser en principe qu'il s'agit d'étudier ici des façons stéréotypées de penser et de sentir. Comme sociologue nous ne nous intéressons pas à ce que X... ou Y... peuvent éprouver en tant qu'individus selon les hasards de leur expérience personnelle. Nous nous intéressons 
seulement à ce qu'ils sentent et pensent en tant que membres d'une communauté humaine »(Malinowski, 1963).

7 Le comportement observé par l'ethnologue n'est pertinent que s'il peut être « relié à la société toute entière dans laquelle il s'inscrit et au sein de laquelle il constitue un système complexe» (Laplantine, 1987). C'est bien ce que nous entendons par observation en perspective à travers laquelle l'action en train de se faire dans sa concrétude n'existe qu'absorbée et écrasée par le modèle culturel, la structure sociale, les représentations culturelles, les mentalités... qui seules intéressent en fin de compte l'option culturaliste de l'ethnologie. La description finale dont l'objectif est d'identifier les éléments caractéristiques de la culture ne peut échapper sans risque à quelques stratégies rhétoriques : la typicalité mentionnant les acteurs sous une appellation générique ou une activité centrale associée à une qualification permanente du personnage, comme si ces dénominateurs communs pouvaient synthétiser l'ensemble des comportements et faisaient oublier les qualités ou les gestes particuliers des uns et des autres. Il serait bon que l'anthropologie du christianisme, qui n'existe pas (ou presque) ne reproduise pas nécessairement les modalités du savoir ethnologique, telles que nous venons de les caricaturer, et si souvent appliquées aux formes religieuses extraeuropéennes.

\section{Pour une anthropologie du fait religieux}

8 L'enjeu de la réflexion proposée ici est bien une représentation de l'homme; nous pourrions dire au sens général du terme : une anthropologie. Nous venons de voir que les grands schèmes des sciences sociales tels qu'ils s'appliquent à l'analyse du fait religieux ne peuvent représenter l'homme concerné autrement qu'en termes :

9 - d'homogénéité insérant l'individu sans statut descriptif ou analytique dans un groupe social qui l'aspire dans son système global aux dépens des particularités circonstancielles, et donnant lieu à des analyses trop classiques sur les identités perdues ou retrouvées ;

10 - de rationalité par laquelle l'homme, en tant qu'acteur social, est capable d'expliciter dans un discours rétrospectif les bonnes raisons qu'il a d'agir et de croire, comme si celles-ci étaient insérées dans un schéma stratégique ou utilitariste ;

11 - de durabilité impliquant une description de l'individu à partir de ses déterminations sociales imprégnant ses gestes et ses paroles comme s'il ne pouvait être qu'en rapport d'adhésion totale et inconditionnelle (selon Bourdieu) avec ceux-ci ;

12 - de signification présentant trop facilement un modèle idéal de l'activité religieuse et de la réception du message associé sous un mode optimal à un maximum d'intensités signifiantes, comme s'il était évident de charger le registre métaphorique (inhérent au religieux) de sens, au risque de la surinterprétation.

13 La vision sociologique ainsi présentée ne semble pas capable de générer un monde possible à vivre. "Si le monde était tel, non seulement comme le décrivent les sociologues, mais aussi, bien souvent, que le rapportent les gens quand ils se mettent dans la disposition de l'“informateur", il serait non seulement invivable mais aussi pas possible à accomplir avec les moyens dont disposent les hommes. » écrit Boltanski (1990). En tout cas, ce mode d'analyse se heurte aux aspérités des scènes courantes de la vie religieuse, telles qu'une observation rapprochée les ferait découvrir. En voici trois exemples rencontrés par hasard au cours d'un tel travail de recherche ${ }^{2}$ : 
Scène 1. Une dame de la soixantaine se prépare à aller à l'église, termine son émission de télévision, s'habille et se dirige vers le lieu de culte. Elle embrasse une amie près de laquelle elle s'assied. Elle écoute, elle s'endort aussi (selon sa propre description) mais ne manque pas la file vers l'hostie. Après le clin d'œil à une autre amie, elle se rassied. Quelques larmes aux yeux. Chez elle, elle ne souffle mot de ce qu'elle a entendu.

Scène 2. La même personne se retrouve le lendemain à une réunion de conseil paroissial. Elle écoute. Plutôt silencieuse. Puis évoque son inquiétude sur l'avenir de l'église dans le village. "Il n'y a plus de curé, les laïcs ne sont pas disponibles et surtout ils ne sont pas formés. Comment voulezvous qu'ils gèrent une inhumation sans prêtre? ». Elle écoute à nouveau. On ne l'entendra plus sur ce sujet. Une prière finale est récitée. Les mains jointes devant elle.

Scène 3. Voici que cette dame participe deux jours plus tard à la préparation de l'assemblée dominicale prochaine (sans prêtre). C'est la première fois. Des questions se posent : le signe de croix, avant ou après l'accueil ? le choix des chants? On lit rapidement le passage concerné de l'Évangile. Et le contenu de la prière universelle? Qui fait quoi? On s'amuse bien, parfois on se tiraille.

17 Sur la base de telles données, cet article n'a pas pour but de présenter des analyses et des résultats sociologiques, mais seulement d'esquisser deux présupposés anthropologiques sans lesquels il est impossible d'imaginer un monde vivable et dont la prise en compte permettrait de repenser la description et l'analyse du fait religieux.

\section{La compétence interactionnelle}

18 Le premier présupposé concerne la compétence interactionnelle des personnes capables de passer, à travers des séquences d'action différentes, de situation en situation et en même temps de se conduire naturellement, selon une disposition conforme à chacun des moments (Boltanski et Thévenot, 1991). Ainsi, la scène l gagnerait beaucoup à une analyse cumulant l'approche interactionnelle classique (à la Goffman, par exemple, mais si peu pratiquée en sociologie du religieux) et une approche représentationnelle tirée des sciences cognitives ${ }^{3}$. De telles recherches sont difficilement possibles sans un ensemble de données photographiques ou filmées et le commentaire sur celles-ci des personnes concernées. Imprégnées d'un ensemble d'incertitudes et de contingences, parce qu'elles sont accomplies par les gens, de telles séquences d'action sont aussi régulées par divers éléments qui sont autant de ressources cognitives pour leur coordination et permettent d'alléger ainsi le processus de réflexion et de délibération pendant leur déroulement : une programmation explicite de la cérémonie, la disposition du lieu de culte (tels objets, à tels endroits) et la présence de différents objets (calice, ciboire...) comme indicateurs de la séquence d'action à exécuter (Piette, 1996b). Cette perspective est d'autant plus intéressante qu'elle permet d'éviter l'analyse surinterprétative associant de manière permanente ces objets à un investissement symbolique ou à des croyances. C'est ici que parallèlement au travail cognitif mis en œuvre pour coordonner ces séquences d'action, ces objets peuvent faire surgir à partir des représentations culturelles qu'ils véhiculent ponctuellement et de manière diversifiées selon les individus, un acte de croire. Un processus de réaction émotionnelle ou d'évocation personnelle (Sperber, 1974) surgit au travers d'une séquence d'action, dans une situation spatio-temporelle précise : l'objet (par exemple l'hostie) devient ponctuellement symbole pour telle personne versant quelques larmes. Il s'agit bien là d'un acte de croire par lequel les dispositions cognitives et affectives d'un individu rejoignent à leur manière les représentations culturelles 
véhiculées ; il adhère, selon différentes modalités (et dont les effets de comportement et d'attitudes sur les autres séquences d'action selon des intensités variables sont possibles mais pas nécessaires), à la représentation mentale ponctuelle soufflée par le contexte situationnel. Comme s'il s'agissait de donner un assentiment selon différents degrés possibles à l'un ou l'autre message évoqué ou objet présenté4.

Ce détour descriptif a, au moins, trois avantages. D'abord il évite le danger d'assimiler l'objet du croire, c'est-à-dire les croyances, aux représentations culturelles du groupe et, en même temps, de faire comme si les représentations mentales propres à un individu constituaient l'exacte réplique des représentations publiques largement diffusées (mythes, dogmes...). « De nombreux anthropologues, de Durkheim à Clifford Geertz, ont considéré, au moins implicitement, que les croyances d'une culture, des plus triviales aux plus mystérieuses, sont mentalement représentées de la même façon et obéissent donc aux mêmes critères de rationalité » (Sperber, 1996). Ensuite l'insistance sur la description des processus interactionnels et cognitifs permet d'éviter l'erreur de considérer comme explicatifs de divers phénomènes collectifs un ensemble de notions (comme celles de symboles ou de croyance, mais on pourrait en ajouter d'autres : identité, sécularisation...) dont la réalité correspondante n'est seulement elle-même qu'une construction ponctuelle restant largement à décrire. Le troisième avantage est capital. La méthode d'observation rapprochée a, en effet, la particularité de mettre le chercheur dans un face à face direct avec les situations dont il fait son objet d'analyse. Il s'agit bien, dans une célébration rituelle (scène 1) ou une réunion administrative de responsables religieux (scène 2), de regarder l'action, les séquences d'action en train de se faire. Au moment de cette observation, il y a bien sûr la terrible interrogation : que regarder et que noter face au détail que le regard doit éliminer pour bien voir et que le texte doit oublier pour être lisible? Ces détails, qui en dissimulent toujours d'autres et sont l'indice de toute la concrétude ordinaire de la vie quotidienne aussi présente dans les situations religieuses, ne peuvent pas être bons à voir pour une « sociologie religieuse » qui refusait à ses débuts (pas très éloignés...) de prendre pour objet l'étude du contenu même de la foi et du dogme chrétien et en même temps pensait que le fait religieux était irréductible à un ensemble de facteurs socioculturels, économiques, politiques, cognitifs ${ }^{5}$... Car regarder le fait religieux en train de se faire dans diverses situations (par exemple, la scène 3), c'est nécessairement repérer que ce contenu ne peut être constitué en noyau dur isolable et explicable après coup par les dits facteurs mais au contraire qu'il n'existe, n'advient et ne se crée en amont que par le jeu ordinaire des pratiques sociales à travers controverses, disputes et contingences bonnes à décrire.

\section{Les limites de la rationalité}

Le deuxième présupposé anthropologique concerne les ressources cognitives limitées par lesquelles l'homme ne peut satisfaire aux normes de non contradiction et de consistance implicitement attribuées aux représentations culturelles et qui l'empêcheraient de soutenir des croyances contradictoires. Notre savoir est faillible et ne peut davantage correspondre à l'omniscience logique telle qu'elle pourrait déduire toutes les conséquences de nos croyances ou désirs et entreprendre les actions pratiques correspondantes (Cherniak, 1986). À quoi s'ajoute un ensemble de difficultés mis en valeur, entre autres analyses, par J. Elster contre les théories du choix rationnel : d'une part un agent n'a pas toujours de préférences entre deux actions, ou il est incapable de les 
comparer; les conséquences incertaines peuvent échapper à son évaluation; il n'existe pas toujours non plus de solution rationnelle dans une interaction; un agent peut difficilement faire, avant de prendre une décision, un calcul rationnel sur l'ensemble des informations dont il dispose. D'autre part, l'agent est aussi influencé par des mécanismes irrationnels : l'incapacité de s'en tenir aux décisions prises (faiblesse de volonté) et la recherche volontaire de ce qui ne peut être obtenu que de façon involontaire (excès de volonté) (Elster, 1986). Ces considérations suffisent à montrer la vanité des bonnes raisons que notre brave dame rencontrée dans les trois scènes ci-dessus pourrait proposer en guise de réponse aux mauvaises questions de l'enquêteur. Il faut absolument éviter de faire des conséquences d'une action le sens et l'intention de celle-ci, comme si l'un et l'autre permettait de la déchiffrer, comme si encore les acteurs sociaux étaient des agents parfaitement rationnels et qu'il ne pouvait pas y avoir dans la vie sociale (religieuse comprise) des accidents dépourvus de sens ou des événements involontaires. C'est ce que nous voulons suggérer à travers les trois scènes en question.

21 Un corollaire direct de cette rationalité limitée est la non-transparence de l'être humain à lui-même et a fortiori aux observateurs. L'homme est toujours ici et ailleurs, en même temps que lui-même et un autre. Et c'est bien dans cette marge de jeu que réside une dimension essentielle de l'adhésion au contenu du discours religieux. Peut-on attendre de l'individu l'adhésion inconditionnelle à des propositions? "Souvent notre rapport aux propositions est tel que nous ne savons tout simplement pas si nous y croyons ou non.» écrit Pavel. "Parfois aussi nous affirmons des propositions auxquelles nous pensons croire; alors qu'en réalité nous y adhérons non pas parce que nous y croyons mais parce que, par exemple, nous admirons la personne de qui nous les tenons. » (Pavel, 1986) Non seulement nous ne pouvons pas attendre une telle adhésion inconditionnelle mais, en plus, de manière très humaine, nous ne pourrons jamais, quelles qu'en soient les garanties, être assurés de la pleine sincérité-authenticité d'une adhésion (Livet, 1994). En deçà de la pleine transparence de l'individu avec lui-même et de son interaction avec les autres, ce qui est en jeu, c'est bien qu' «il ne fait en dernière instance qu'affirmer son adhésion à la double ontologie dans laquelle le niveau sacré s'articule vers l'univers profane. La conscience religieuse repose sur des modèles ontologiques disposant de deux réseaux de références aussi distincts que possible et néanmoins étroitement liés » (Pavel, 1986). Le prêtre qui... c'est aussi un homme... c'est la possibilité pour l'homme de croire et de ne pas croire en même temps à son propre jeu, de se situer en tout cas entre la sincérité et le cynisme dans « une sorte de point intermédiaire où l'on peut se tenir au prix d'une relative lucidité sur soi» (Goffman, 1973). Entre la scène impossible où se déroulerait la représentation selon des normes idéales et les coulisses, tout aussi impossibles, qui impliquent une latitude de contredire l'impression produite par les représentations, il n'existe que des cas hybrides mêlant les deux registres et laissent des traces d'un effet d'humanité : tourner sa tête, jeter un regard distrait, avoir l'air absent, entamer un court aparté avec son voisin (cf. la scène 1). Bref, contingences et particularités selon un dosage humain d'attention et de souplesse intérieure pour réguler toute situation. Le religieux en train de se faire n'en est pas dépourvu comme en témoignerait une description précise des scènes 2 et 3.

22 À notre sens, le rituel, comme celui de la célébration dominicale où participe notre dame, cristallise parfaitement cette non-transparence de l'homme. En même temps qu'il affirme la fictionalité de ce qu'il dit et de ce qu'il fait, il en exprime, et sans doute à la différence du jeu proprement dit, l'extrême importance. Les gens ont leur raison d'accorder leur 
confiance au prêtre dont ils reconnaissent la compétence particulière et la place déterminante qu'il occupe dans la tradition de l'Église, etc. Dans le rituel religieux, ce n'est pas un Crétois qui dit que tous les Crétois sont des menteurs; c'est un homme crédible qui ne peut dissimuler la fictionalité de ses gestes et de ses paroles, tout en se donnant du mal pour montrer leur importance... mais sans éviter l'autoné-gation implicite. Le rituel incite aussi à l'interrogation nécessairement sans réponse : est-ce du sérieux ? n'est-ce que du spectacle? est-ce la réalité ? la fiction? La réussite du rituel se trouve dans l'oscillation infinie entre ces questions. Une réponse à l'un des pôles menacerait la répétitivité du rituel. Aux participants de jouer ou de ne pas jouer le jeu du rituel, de se laisser ou de ne pas se laisser prendre à ses pièges : croire vraiment que $\mathrm{X}$, croire que $\mathrm{X}$ est vraiment, croire quand même que $\mathrm{X}$, ne pas croire vraiment que $\mathrm{X}$, ne pas croire du tout... (Piette, 1996c). Il y a bien plusieurs modalités d'adhésion : être convaincu que c'est la réalité, jouer à faire comme si...(mais à fond), ou encore ne pas jouer à fond à faire comme si...

\section{Pour ne pas conclure...}

Cet article ne veut pas aller plus loin dans le traitement des données à recueillir au cours d'un travail d'observation rapprochée. Il se donnait seulement deux objectifs. D'abord la critique des modalités d'observation du fait religieux en sciences sociales et, de là, des schèmes sous-tendant la construction et la représentation de l'objet sociologique et de la vision du monde qui en découle. Ensuite, et commençant à faire le chemin inverse, il se proposait de partir de deux présupposés anthropologiques inséparables d'une vision du monde qui soit vivable par les êtres humains et de montrer comment ces présupposés pouvaient orienter une description et une analyse différentes du fait religieux. Il reste cependant à confronter ces objectifs théoriques, le plus souvent nourris des avancées récentes de la sociologie de l'action, aux données de terrain...

\section{BIBLIOGRAPHIE}

BAzIN Jean, « Les fantômes de Mme Du Deffand : exercices sur la croyance », Critique, n 529-530, 1991, pp. 492-511.

BOLTANSKI Luc, L'Amour et la justice comme compétence, Paris, Métailié, 1990.

BOLTANSKI Luc et THÉVENOT Laurent, De la justification, Paris, Gallimard, 1991.

CHERNIAK C, Minimal Rationalité, Cambridge, The MIT Press, 1986.

ClAVERIE Elisabeth, « La Vierge, le désordre, la critique », Terrain, 14, 1990, pp. 60-75.

ConEIN Bernard, « Cognition située et coordination de l'action » Réseaux (CNET), n 43, 1990, pp. 100-110.

DURKHeIM Émile, Les règles de la méthode sociologique, Paris, PUF, (7éd.), 1993. 
ELSTER Jon, Le laboureur et ses enfants, Paris, Minuit, 1986.

GoffMAN Erving, La mise en scène de la vie quotidienne (T. 1), Paris, Éditions de Minuit, 1973.

HERVIEU-LÉGER Danièle, La religion pour mémoire, Paris, Cerf, 1993.

LAPLANTINE François, Clefs pour l'anthropologie, Paris, Seghers, 1987.

LIVET Pierre, La communauté virtuelle. Action et communication, Combas, L'Éclat, 1994.

MaLINOWSKI Bronislaw, (1922), Les Argonautes du Pacifique Oriental, Paris, Gallimard, 1963.

PAVEL Thomas, Univers de fiction, Paris, Seuil, 1986.

PIETTE Albert, Ethnographie de l'action, Paris, Métailié, 1996 a.

PIETTE Albert, «L'institution religieuse en images. Modèle de description ethnographique ».

Archives de Sciences Sociales des Religions, $\mathrm{n}^{\circ}$ 93, 1996 b, pp. 51-80.

PIETTE Albert, « Les hommes et leurs croyances » in L. voYÉ (éd.), Figures des Dieux, Bruxelles, De Boek, 1996 c.

SPERBER Dan, Le symbolisme en général, Paris, Hermann, 1974.

SPERBER Dan, La contagion des idées, Paris, Odile Jacob, 1996.

WeBER Max, Essai sur la théorie de la science, Paris, Plon, 1965.

Weber Max, (1921) Économie et Société, tome 1, Paris, Plon, 1971.

\section{NOTES}

1. Il constitue la version écrite d'une communication présentée à la journée d'étude « Les formes sensibles de la religion » en juin 1996. Elle présentait alors un programme de recherche dont la réalisation a donné lieu à un livre La religion de près, l'activité religieuse en train de se faire, Métailié, 1999.

2. Il ne s'agit pas ici de détailler le contexte de cette recherche ni de préciser les situations desquelles ces trois scènes ont été extraites. Nous renvoyons à l'ouvrage cité infra.

3. Voir les travaux de B. Conein (1990, par ex.) sur cette double approche appliquée bien sûr à d'autres domaines que le religieux.

4. Cf. parmi les travaux d'anthropologie du croire : BAZIN, 1991 et CLAVERIE, 1990. Cf. également P IETTE, 1996c.

5. Voir à ce sujet les remarques de Danièle HERVIEU-LÉGER, 1993.

\section{RÉSUMÉS}

Cet article se donne seulement deux objectifs :

- la critique des modalités d'observation du fait religieux en sciences sociales, des schèmes qui sous-tendent la construction et la représentation de l'objet sociologique et de la vision du monde qui en découle ; 
- préconisant le chemin inverse, il propose de partir de deux présupposés anthropologiques inséparables d'une vision du monde qui permette de rendre compte de l'activité d'êtres humains : la compétence interactionnelle et les limites de la rationalité ; et de montrer comment ses présupposés pourraient orienter une description et une analyse différentes du fait religieux. Il reste à confronter ces objectifs théoriques, le plus souvent nourris des avancées récentes de la sociologie de l'action, aux données de terrain...

This article has two limited goals. The first one is to criticize the modalities of observation of the religious factor by the social sciences, the schemas that underlie the construction and the representation of the sociological object, and the vision of the world they entail. The author recommends a different approach which starts from two anthropological presuppositions inseparable from a vision of the world that makes it possible to account for the activity of human beings: interactional competence and the limits of rationality. The second goal is to show how these presuppositions could lead to a different description and analysis of the religious factor. Of course, these theoretical goals, which have been influenced by recent developments in the sociology of action, should now be tested empirically...

Este artículo se plantea dos objetivos : en primer lugar, propone una crítica de las modalidades de observación del hecho religioso en las ciencias sociales, de los esquemas que fundan la construcción y la representación del objeto sociológico, y de la vision del mundo que deriva de ello ; en segundo lugar, preconiza caminar en el sentido contrario y partir de dos presupuestos antropológicos inseparables de una visión del mundo que permite dar cuenta de la actividad de los seres humanos: la competencia interaccional y los limites de la racionalidad: en otros términos, pretende mostrar cómo estos presupuestos podrían orientar una descripción y un análisis diferentes del hecho religioso. Falta todavía la confrontación de estos objetivos teóricos, enriquecidos cada vez más por los avances recientes de la sociología de la acción, con los datos de campo...

\section{AUTEUR}

\section{ALBERT PIETTE}

Université de Paris VIII, CEIFR - CNRS/EHESS 